\title{
The Validation Index: A new metric for validation of segmentation algorithms using two or more expert outlines with application to radiotherapy planning
}

Validation is required to ensure automated segmentation algorithms are suitable for radiotherapy target definition. In the absence of true segmentation, algorithmic segmentation is validated against expert outlining of the region of interest. Multiple experts are used to overcome inter-expert variability. Several approaches have been studied in the literature, but the most appropriate approach to combine the information from multiple expert outlines, to give a single metric for validation, is unclear. None consider a metric that can be tailored to case-specific requirements in radiotherapy planning. Validation index (VI), a new validation metric which uses the experts' level of agreement was developed. A control parameter, $\alpha$, was introduced for the validation of segmentations required for different radiotherapy scenarios: for targets close to organs-at-risk and for difficult to discern targets, where large variation between experts is expected. VI was evaluated using two simulated idealised cases and data from two clinical studies. VI was compared with the commonly used Dice similarity coefficient $\left(\mathrm{DSC}_{\text {pair-wise }}\right)$ and found to be more sensitive than the $\mathrm{DSC}_{\text {pair-wise }}$ to the changes in agreement between experts. VI was shown to be adaptable to specific radiotherapy planning scenarios.

\section{Introduction}

Radiotherapy planning requires segmentation of target and normal tissue structures. Mostly, this is performed manually on two-dimensional slices by experts and is time consuming. Wider use of adaptive radiotherapy (ART) has increased the need for automated segmentation algorithms [1-6]. Validation of these algorithms is important to assess their suitability for the clinic. Expert opinion, i.e., manual outlining of the region of interest (ROI) is commonly used for validation. Outlines from two or more experts are used to overcome inter-expert variability, i.e., the uncertainty in ground truth. Figure 1(a) illustrates a case in which an algorithmic segmentation (thick line) is validated by comparison with two experts' outlines. Several regions may be defined: where the algorithm agrees 
with both experts (1), one expert (2), and neither expert (3) and where experts agree but the algorithm does not (4). How should these agreements and differences amongst expert outlines be used for quantitative validation?

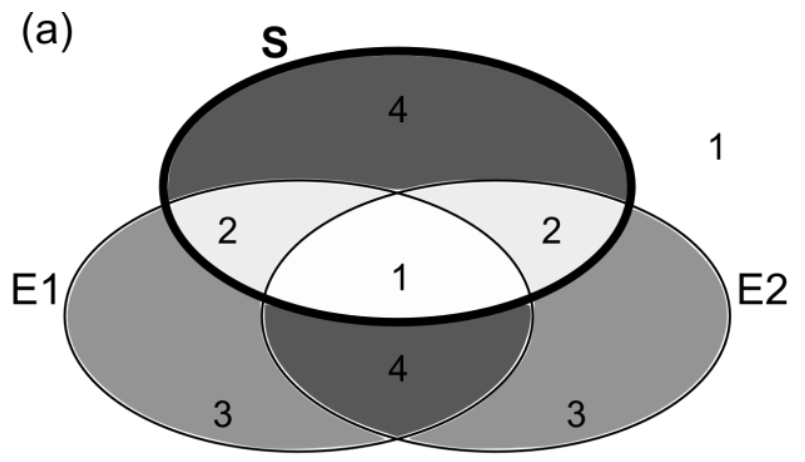

(b)

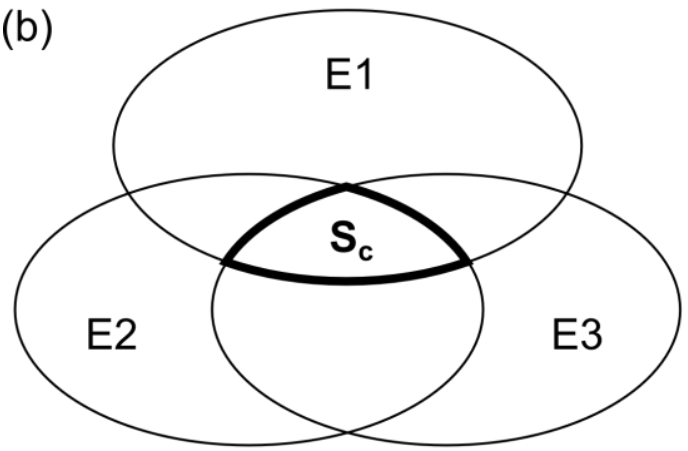

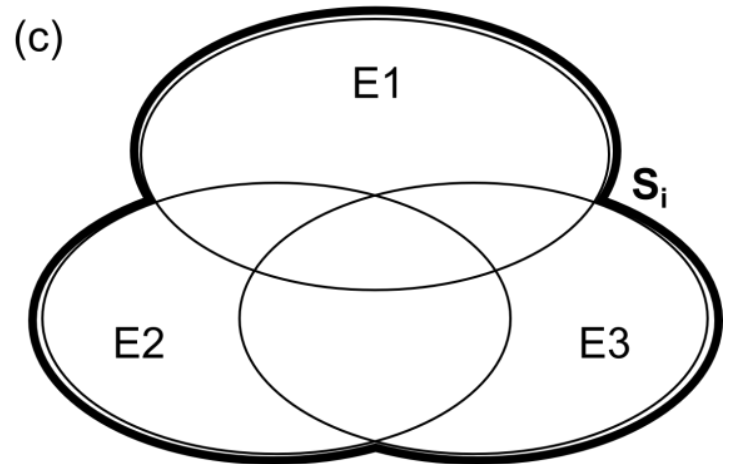

Figure 1. Validation of algorithmic segmentation by multiple experts. (a) A segmentation $\mathrm{S}$ validated by 2 expert outlines E1 and E2. Different regions can be identified, where the algorithm agrees with both experts (1), one expert (2), and neither expert (3) and where experts agree but the algorithm does not (4), (b) shows an example segmentation $S_{c}$ and (c) shows an example segmentation $S_{i}$. Both $S_{c}$ and $S_{i}$ are for the same ROI validated by 3 expert outlines E1, E2 and E3.

The Dice similarity coefficient (DSC) [7], the ratio of the volume of intersection of two segmentations to their mean volume, is a commonly used metric for the validation of automated segmentation algorithms in radiotherapy [1-6]. Often, in the case of multiple outlines, the DSC is calculated for an ROI (i.e., the segmentation) and each expert outline individually. DSCs are then averaged over all outlines. This metric will be referred to as $\mathrm{DSC}_{\text {pair-wise. }}$. It does not use the knowledge of expert agreements and disagreements which becomes important in the context of target definition for radiotherapy planning. Segmentation of radiotherapy volumes should be tailored to case-specific clinical requirements. For example, in the case of a target close to an organ-at-risk (OAR), to 
minimise dose to the OAR, a conservative segmentation algorithm is required (see fig 1(b)). Validation of this algorithm should penalise segmentation of regions in which few experts' agree. In the case of target which is difficult to discern such as breast tissue, an inclusive segmentation algorithm may be required. To avoid under-dosage to the target, validation should consider all the regions of experts outline (see fig 1(c)).

An approach, that incorporates the uncertainty in ground truth, is to perform validation based on the regions (see fig 1(a)) with different levels of agreement between multiple experts. This study proposes to evaluate segmentation algorithm performance by measuring spatial overlaps of algorithmic segmentation with these regions, i.e., levels of agreement between multiple experts. Spatial overlaps with regions where fewer experts agree were penalised because it is less likely that they represent ground truth. These spatial overlap measures were weighted and summed up to report a single evaluation metric.

Kouwenhoven et al. [8] presented a conformity index to measure the similarity amongst manual outlines. A Sum Rule [9] approach was used to combine levels of agreement between experts. It did not consider the validation of a single region (algorithmic segmentation) with expert outlines, nor did it consider specific clinical requirements.

The motivation for this work was to develop a method for validating segmentation of radiotherapy treatment plans that incorporates the opinions of several experts.

Our aim was to develop the validation index (VI), a single figure-of-merit, for validation of algorithmic segmentation of ROIs used in radiotherapy planning that uses the level of agreement between multiple experts. Herein, algorithmic segmentation is referred to as segmentation. VI can be adapted to the specific radiotherapy requirements by adjusting the importance of the regions for which greater numbers of experts agree. It was developed by constructing a reference standard from multiple expert outlines and adapting the DSC to measure the conformity between the segmentation and levels of agreement within the reference standard. The use of VI to validate segmentation is demonstrated using simulated and clinical datasets. Robustness of VI to the number of experts and its use in specific radiotherapy planning scenarios was evaluated. 


\section{Methods and Materials}

The analysis presented here can be used for $2 \mathrm{D}$ and $3 \mathrm{D}$ data, using pixels and voxels respectively. In the text, points refer to representing either the pixels or voxels. In this section the derivation of VI is presented. The simulated and clinical datasets, used to evaluate VI, are described.

\subsection{Description of the Validation Index}

To develop a validation metric, a multi-expert reference standard was built using multiple expert outlines and the levels of agreement between experts. The reference standard represents the best estimate of the ground truth, i.e., the true segmentation. Experts' level of agreement was defined by the volume of overlap, $V_{k}$, the volume formed by the agreement of $k$ experts (see figure 2a). The concept of membership value $(m)$ was introduced for these volumes of overlap $V_{k}$, defined as the proportion of agreeing experts, $m=k / N$. Where $k$ was the number of experts agreeing and $N$ was the total number of experts. Membership value, $m$, represents the probability that a volume of overlap is ground truth, $m=1$ when all experts agree and reduces as fewer experts are in agreement.

Next, the Dice similarity coefficient (DSC) was adapted to validate the segmentation with individual volumes of overlap $\left(V_{k}\right)$ of the reference standard. Validation was performed iteratively. For $N$ expert outlines, whole segmentation, $S$, was compared with $V_{N}$ (the volume for which all experts agree). The partial segmentation, $S_{N-1}$, (the portion of S not coincident with $V_{N}$ ), was compared with $V_{N-1}$. The partial segmentation $S_{N-2}$, (the portion of $S_{N-1}$, not coincident with $V_{N-1}$ ), was compared with $V_{N-2}$. And so on. Segmentation $S_{k}$, which is validated by $k$ experts, is defined by:

$$
S_{k}=\left\{\begin{array}{rlrl}
S & k & =N \\
S_{k+1}-\left(V_{k+1} \cap S\right) & k & \in[N-1,1]
\end{array}\right.
$$

For each volume of overlap $V_{k}, D S C(k)$ was calculated using:

$$
D S C(k)=2 \frac{\left|V_{k} \cap S_{k}\right|}{\left|V_{k}\right|+\left|S_{k}\right|} .
$$

The validation measure $(V M)$ of a specific point was defined as the product of its membership value $m$ and $D S C(k)$, given by: 


$$
V M=m D S C(k)=2 \frac{k}{N} \frac{\left|V_{k} \cap S_{k}\right|}{\left|V_{k}\right|+\left|S_{k}\right|} .
$$

The set of validation measures gives a description of the conformity between the segmentation and experts' outlines. VMs can be averaged in order to report the validation results using a single "figure-of-merit". A weight parameter $(w)$ for the validation measures was introduced. Intuitively, weights should be greatest when all experts agree and reduce as fewer experts agree. Weights were defined using the volumes of experts' agreements $\left(V_{k}\right)$, and are given by:

$$
w_{k}=\frac{k^{\alpha}\left|V_{k}\right|}{\sum_{j=1}^{N} j^{\alpha}\left|V_{j}\right|}
$$

where, $\alpha$ is control parameter introduced to allow adjustment of the weights according to specific radiotherapy planning requirements.

Validation index (VI) is the weighted average of the validation measures, given by:

$$
V I=\sum_{k=1}^{N} w_{k} m D S C \quad(k)=\sum_{k=1}^{N}\left|\frac{k^{\alpha}\left|V_{k}\right|}{\sum_{j=1}^{N} j^{\alpha}\left|V_{j}\right|}\right|\left(\frac{k}{N}\right)\left(2 \frac{\left|V_{k} \cap S_{k}\right|}{\left|V_{k}\right|+\left|S_{k}\right|}\right)
$$

Control parameter $\alpha$ can be set to zero or any positive value. For $\alpha=0$, all membership values have weight equal to their fractional volumes in the reference standard. Higher values will increase the weight of the highest membership values. For $\alpha=1, w$ describes a normalized frequency at which the membership values $(m)$ occur for a given set of outlines. An illustration of a 3-expert reference standard and how validation is performed is illustrated in figure 2 . 


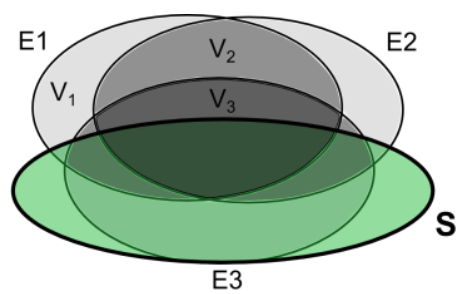

a. Ref. standard $\left(V_{3}, V_{2}\right.$, and $\left.V_{1}\right)$ and Segmentation (S)

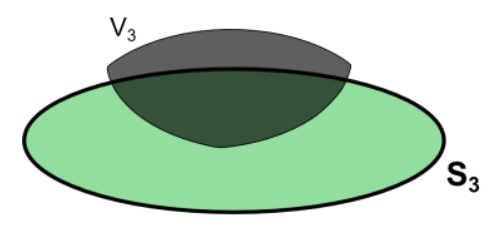

b. Validation at level $3(m=1)$ :

$70 \%$ of $V_{3}$ coincides with $S_{3}$

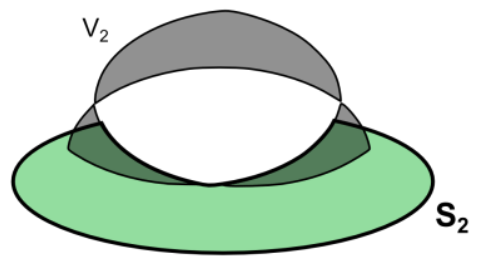

c. Validation at level $2(\mathrm{~m}=0.67)$ : $60 \%$ of $V_{2}$ coincides with $S_{2}$

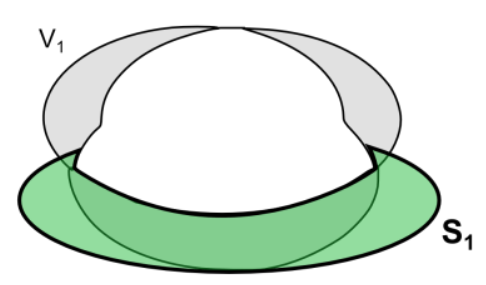

d. Validation at level $1(m=0.33)$ : $50 \%$ of $V_{1}$ coincides with $S_{1}$

Figure 2. Validation of segmentation $(S)$ by three expert outlines E1, E2 and E3; a. Reference standard formed by 3 experts, experts' level of agreement is represented by the volumes of overlap: $V_{3}$ (all experts agree, $m=1$ ), $V_{2}$ (two experts agree, $m=0.67$ ), and $V_{1}$ (no expert agreement, $m=0.33$ ); b. Validation by $V_{3}$; c. Validation by $V_{2}$; d. Validation by $V_{l}$.

\subsection{Datasets}

Two simulated, idealised datasets (A and B) and two clinical datasets (C and D) were used to evaluate VI. The first, A, comprised 3 cases, each consisting of 3 expert outlines and a segmentation, each have equal volume (V), see figure 3. These cases varied from each other only in terms of the volumes of overlap $V_{3}, V_{2}$ and $V_{1}$. The second dataset, B, comprised two cases designed to illustrate how VI can be adapted for specific radiotherapy planning requirements. These were conservative segmentation, $\mathrm{S}_{\mathrm{c}}$, (case B1) and inclusive segmentation, $\mathrm{S}_{\mathrm{i}}$, (case B2). As for dataset $\mathrm{A}$, the reference standard was formed by three idealised expert outlines each with volume $(\mathrm{V}) . \mathrm{S}_{\mathrm{c}}$ was equal to the intersection of 3 expert outlines $\left(V_{3}\right)$, and $\mathrm{S}_{\mathrm{i}}$ was equal to the union of the expert outlines $\left(V_{3} \cup V_{2} \cup V_{1}\right)$. Table 1 gives the details of the volumes of overlap and corresponding percentage overlaps with the segmentation, for datasets A and B. Clinical datasets were obtained from two algorithm validation studies. In both studies 2D slices were used for validation. Dataset C compared different algorithms for segmentation of fibroglandular breast tissue from computed tomography (CT) images [10]. Three experts (one clinical oncologist and two radiologists) outlined the fibroglandular tissue on 12 mid breast CT images (from three patients). An example of expert outlines and the corresponding 
reference standard is shown in figure 3(a) and 3(b), respectively. Our previous study [11] showed that the fuzzy c-means method with three classes (fat, fibroglandular, and background) gives accurate breast tissue segmentation and therefore this method was used to generate segmentations for each of the 12 slices. Clinical dataset D was generated by Kaus et al. [12,13] to validate an automated brain tissue segmentation method. Four experts outlined brain, tumour, ventricles, and necrosis on 10 magnetic resonance images (MRI, one from each of 10 patients). In the present study, VI was calculated for segmentation of brain tissue and tumour, a total of 20 outlines. An example of experts' outlines of brain tissue, and the corresponding reference standard is shown in figure 3(c) and 3(d), respectively.

Table 1. Description of the two simulated datasets used for analysis of validation index (VI).

\begin{tabular}{|c|c|c|c|c|c|c|c|c|}
\hline \multirow{3}{*}{ Dataset } & \multirow{3}{*}{ Case } & \multicolumn{4}{|c|}{ Component parts of the } & \multicolumn{3}{|c|}{ \% Ref. Std. overlaps } \\
\hline & & \multicolumn{4}{|c|}{ Reference Standard (Ref. Std.) } & \multicolumn{3}{|c|}{ with segmentation } \\
\hline & & $\mathrm{V}_{3}$ & $\mathrm{~V}_{2}$ & $\mathrm{~V}_{1}$ & Total & $\left(\mathrm{V}_{3}, \mathrm{~S}_{3}\right)$ & $\left(\mathrm{V}_{2}, \mathrm{~S}_{2}\right)$ & $\left(\mathrm{V}_{1}, \mathrm{~S}_{1}\right)$ \\
\hline \multirow[t]{3}{*}{$\bar{A}$} & Case A1 & $0.75 \mathrm{~V}$ & $0.25 \mathrm{~V}$ & $0.25 \mathrm{~V}$ & $3.0 \mathrm{~V}$ & $90 \%$ & $60 \%$ & $50 \%$ \\
\hline & Case A2 & $0.80 \mathrm{~V}$ & $0.20 \mathrm{~V}$ & $0.20 \mathrm{~V}$ & $3.0 \mathrm{~V}$ & $90 \%$ & $60 \%$ & $50 \%$ \\
\hline & Case A3 & $0.85 \mathrm{~V}$ & $0.15 \mathrm{~V}$ & $0.15 \mathrm{~V}$ & $3.0 \mathrm{~V}$ & $90 \%$ & $60 \%$ & $50 \%$ \\
\hline \multirow[t]{2}{*}{ B } & Case B1 & $0.1 \mathrm{~V}$ & $1.3 \mathrm{~V}$ & $0.1 \mathrm{~V}$ & $3.0 \mathrm{~V}$ & $100 \%$ & $0 \%$ & $0 \%$ \\
\hline & Case B2 & $0.1 \mathrm{~V}$ & $1.3 \mathrm{~V}$ & $0.1 \mathrm{~V}$ & $3.0 \mathrm{~V}$ & $100 \%$ & $100 \%$ & $100 \%$ \\
\hline
\end{tabular}

Note: In all cases, 3 expert outlines and segmentation have equal volume $(\mathrm{V}) ; \mathrm{V}_{\mathrm{k}}$ represents the volume formed by the agreement of $k$ experts; $\left(\mathrm{V}_{\mathrm{k}}, \mathrm{S}_{\mathrm{k}}\right)$ represents the percentage of $\mathrm{V}_{\mathrm{k}}$ that coincides with the $\mathrm{S}_{\mathrm{k}}$; Total = $3 \mathrm{~V}_{3}+2 \mathrm{~V}_{2}+1 \mathrm{~V}_{1}$. 


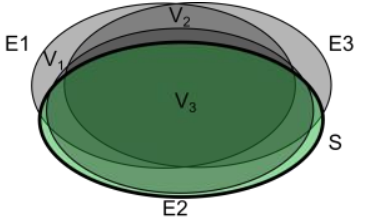

Case A1. Reference standard: $\mathrm{V}_{3}=0.75 \mathrm{~V}, \mathrm{~V}_{2}=0.25 \mathrm{~V}$ $\mathrm{V}_{1}=0.25 \mathrm{~V}$; and Segmentation: $\mathrm{S}=\mathrm{V}$

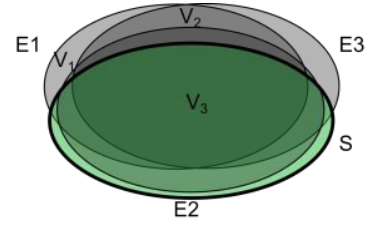

Case A2. Reference standard: $\mathrm{V}_{3}=0.80 \mathrm{~V}, \mathrm{~V}_{2}=0.20 \mathrm{~V}$, $\mathrm{V}_{1}=0.20 \mathrm{~V}$; and Segmentation: $\mathrm{S}=\mathrm{V}$

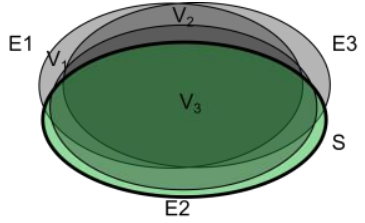

Case A3. Reference standard: $\mathrm{V}_{3}=0.85 \mathrm{~V}, \mathrm{~V}_{2}=0.15 \mathrm{~V}$, $\mathrm{V}_{1}=0.15 \mathrm{~V}$; and Segmentation: $\mathrm{S}=\mathrm{V}$

Figure 3. Dataset A: In all the three cases (A1, A2, A3), a segmentation (S) was validated against the reference standard formed by three experts (E1, E2, and E3), experts level of agreement is represented by the volumes of overlap: $V_{3}$ (all experts agree, $\left.m=1\right), V_{2}$ (two experts agree, $m=0.67$ ), and $V_{1}$ (no expert agreement, $m=0.33$ ). From case A1 to A3, the volume of agreement of all three experts $\left(V_{3}\right)$ increases. 


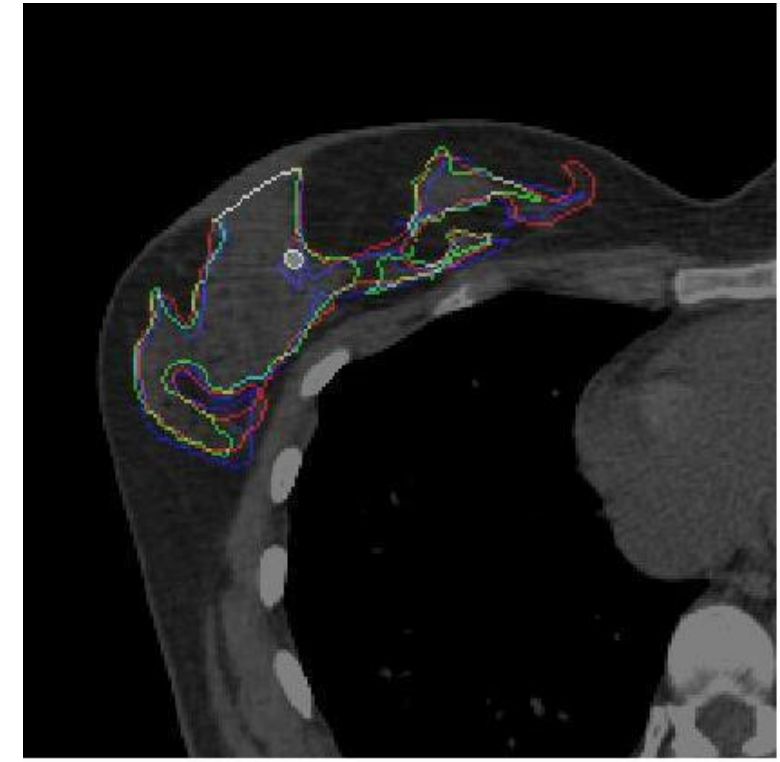

a. Fibroglandular tissue outlining

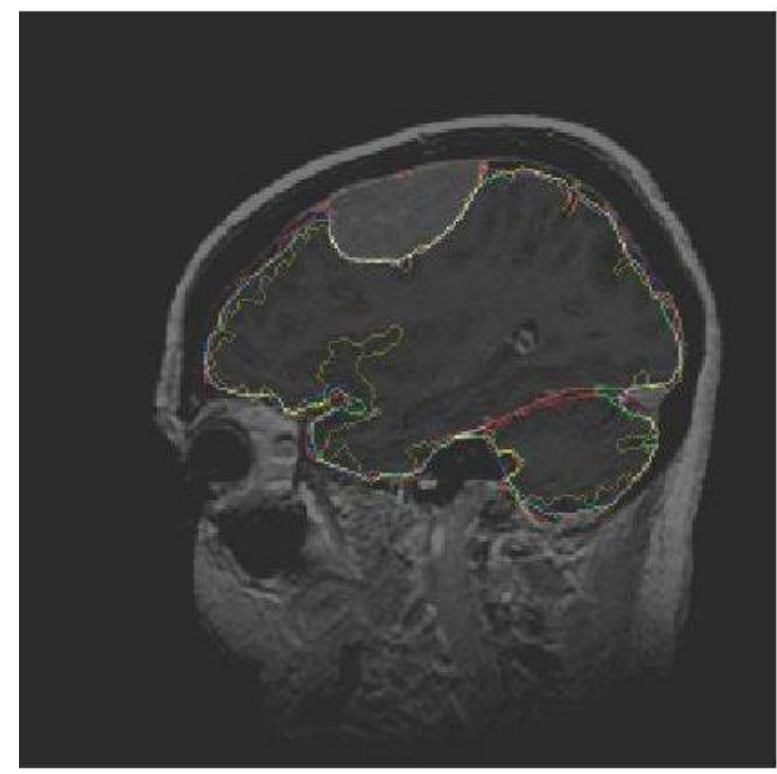

c. Brain tissue outlining

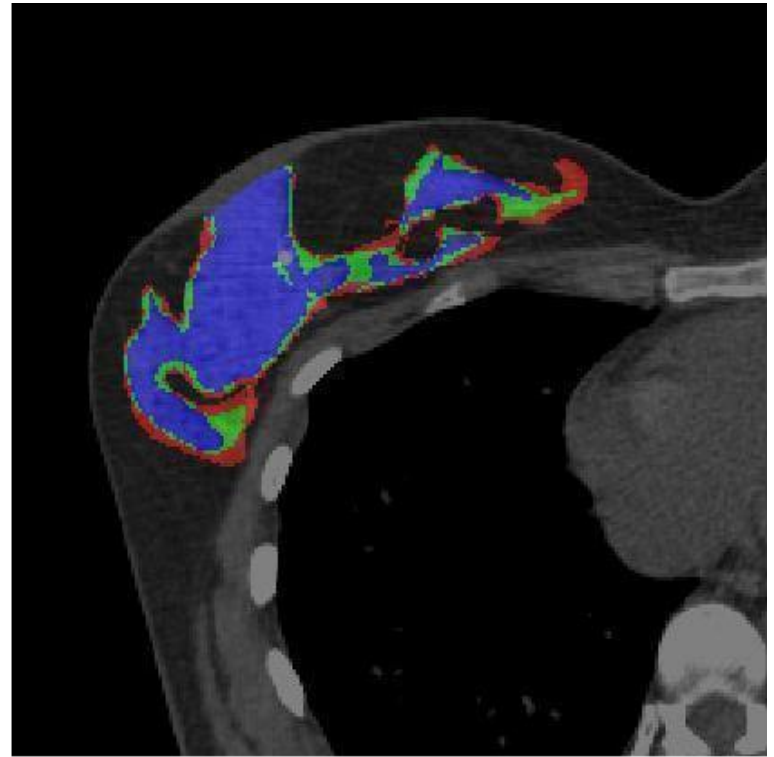

b. Fibroglandular tissue reference standard

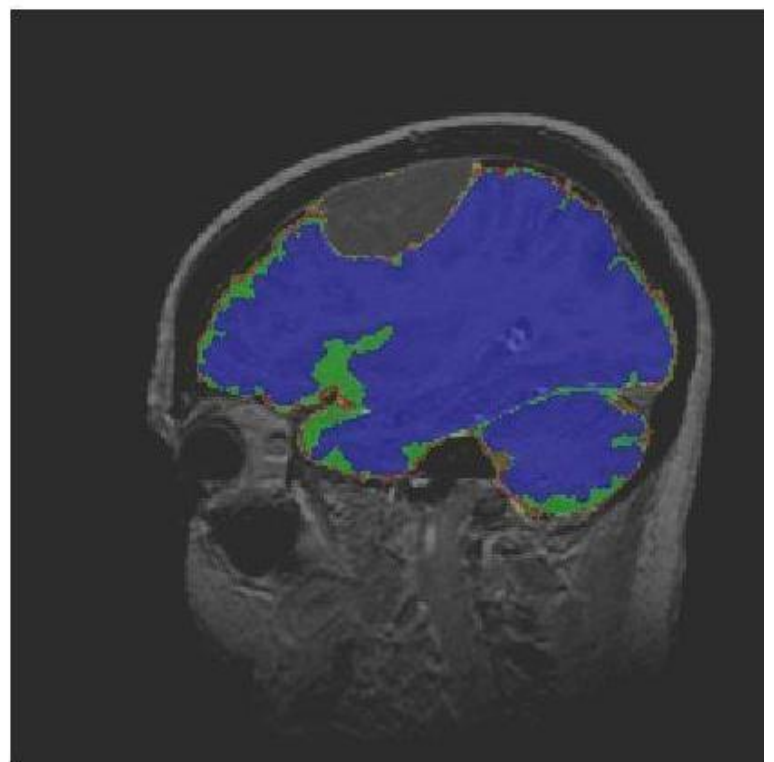

d. Brain tissue reference standard

Figure 3. Examples of experts' outlines and levels of agreement. (a) Three fibroglandular tissue outlines on axial CT images; (b) A reference standard constructed from outlines shown in (a): blue $\left(V_{3}\right)$, green $\left(V_{2}\right)$, and red $\left(V_{l}\right)$; (c) Four brain tissue outlines on sagittal MR images; (d) A reference standard constructed from outlines shown in (c): blue $\left(V_{4}\right)$, green $\left(V_{3}\right)$, red $\left(V_{2}\right)$, and yellow $\left(V_{1}\right) . V_{k}$ represents the volume formed by the agreement of $k$ experts. 


\subsection{Evaluation of VI}

The VI and $\mathrm{DSC}_{\text {pair-wise }}$ (DSC $\mathrm{Dair}_{\text {-wise }}$ as defined in the introduction, is an average of DSCs of all individual outlines) were evaluated and compared for simulated dataset $\mathrm{A}$ and the two clinical datasets $\mathrm{C}$ and $\mathrm{D}$. The effect of the control parameter $\alpha$ on the validation index was investigated for all datasets. For each dataset VI was calculated with $\alpha=0,1,2,3,4,5,6,7,8$, 9, and 10. VI for indicative radiotherapy planning requirements was evaluated by comparing VI as a function of $\alpha$ for a conservative segmentation and an inclusive segmentation i.e., case B1 and case B2, respectively. The effect of number of experts on VI and $\mathrm{DSC}_{\text {pair-wise }}$ was analysed using datasets $\mathrm{C}$ and $\mathrm{D}$. Variations in VI with different number of experts may be influenced by variation between experts. Inter-expert variations were measured for both clinical datasets using DSC. VI and $\mathrm{DSC}_{\text {pair-wise }}$ were calculated using all experts, (this was called the primary group) and excluding one expert at a time from the primary group. Analysis of variance (ANOVA) was used to test for statistical differences between VI calculated using the primary group (all experts) and the groups with one expert removed.

\section{Results}

\subsection{Comparison of VI and DSC $C_{\text {pair-wise }}$}

VI and $\mathrm{DSC}_{\text {pair-wise }}$ for datasets $\mathrm{A}, \mathrm{C}$ and $\mathrm{D}$ are given in Table 2. Both $\mathrm{VI}$ and $\mathrm{DSC}_{\text {pair-wise }}$ increased with increasing agreement $\left(\mathrm{V}_{3}\right)$ between the three experts in the idealised cases $\mathrm{A} 1, \mathrm{~A} 2$ and $\mathrm{A} 3$. For these three cases, the relative change in the metrics from case to case (i.e. increasing agreement, $V_{3}$ ) was higher for VI than the $\mathrm{DSC}_{\text {pair-wise }}$. This suggests VI was more sensitive than $\mathrm{DSC}_{\text {pair-wise }}$ to the changes in agreement between the experts. For all datasets, VIs were smaller than $\mathrm{DSC}_{\text {pair-wise, }}$, this will be further considered in discussion section. 
Table 2. Validation metrics (VI and $\mathrm{DSC}_{\text {pair-wise}}$ ) for simulated dataset $\mathrm{A}$, and mean (and standard deviation) of the validation metrics for clinical dataset $\mathrm{C}$ and $\mathrm{D}$.

\begin{tabular}{lcc}
\hline \hline & VI $\alpha=1$ & DSC $_{\text {pair-wise }}$ \\
\hline Case A1 $\left(\mathrm{V}_{3}=0.75\right)$ & 0.65 & 0.82 \\
Case A2 $\left(\mathrm{V}_{3}=0.80\right)$ & 0.70 & 0.83 \\
Case A3 $\left(\mathrm{V}_{3}=0.85\right)$ & 0.74 & 0.85 \\
Dataset C (Breast)* $(\mathrm{n}=12)$ & $0.68(0.06)$ & $0.80(0.06)$ \\
Dataset D (Brain tissue $) *(\mathrm{n}=10)$ & $0.82(0.09)$ & $0.92(0.04)$ \\
Dataset D (Brain tumour)* $(\mathrm{n}=10)$ & $0.77(0.12)$ & $0.88(0.09)$
\end{tabular}

Note: $\mathrm{V}_{3}$ represents the volume of overlap of all three experts; VI is validation index; DSC is Dice similarity coefficient.

\subsection{Effect of control parameter, $\alpha$}

Figures 4 (a) and 4 (b) give VI as a function of $\alpha$ for dataset A and datasets C and D, respectively. For these datasets, VI increases with the increasing control parameter $(\alpha)$. This is because the volume of overlap with highest membership value $(m=1)$ has the largest spatial overlap (DSC) with the segmentation and for higher weights the highest membership value gets more weighting. For $\alpha>5$ only the volumes with the highest agreement contribute noticeably to the VI and therefore VI asymtotes towards a maximum value of VI.

The validation index for two different segmentation cases, case B1 and B2, see figure 1(a) and (b), as a function of control parameter $(\alpha)$ is presented in figure 4(c). For the conservative segmentation $\mathrm{S}_{\mathrm{c}}$ (case B1), the behaviour of VI is same as for the clinical cases i.e., VI increases with increasing $\alpha$. For the inclusive segmentation $\mathrm{S}_{\mathrm{i}}$ (case B2), VI decreases with increase in $\alpha$. In this case, the volume of overlap with $m=1$ has lower overlap (DSC) with the segmentation, compared to the volume with the next lowest membership value, $m=0.67$. Increasing $\alpha$ increases the contribution from volumes with higher membership values and therefore VI decreases in this case. For a conservative segmentation algorithm greater weigh $(\alpha=10)$ should be used. For an inclusive segmentation algorithm lower weight ( $\alpha=0$ or 1 ) should be used. 

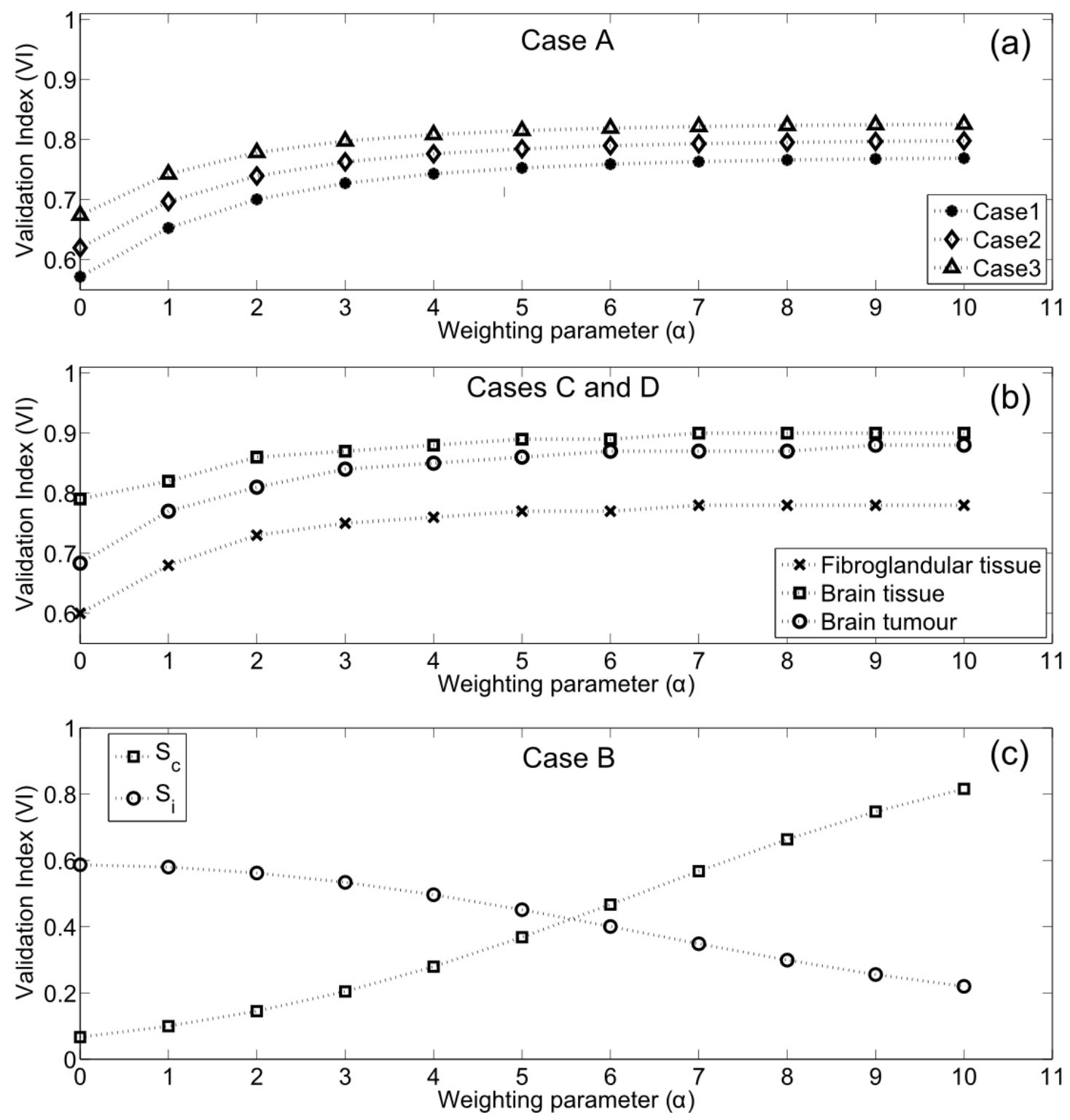

Figure 4. a) VI as a function of $\alpha$ for cases A1, A2 and A3. b) mean VI as a function of $\alpha$ for datasets C and D.

c) VI as a function of $\alpha$ for a conservative segmentation, $\mathrm{S}_{\mathrm{c}}$ (case B1) and an inclusive segmentation, $\mathrm{S}_{\mathrm{i}}$ (Case B2).

\subsection{Variation between experts}

Figure 5(a) and (b) shows the DSC for the segmentation and each expert outline of fibroglandular breast tissue and brain tissue, respectively. The variation between expert outlines of the same structure varied between cases. The inter-expert variation for brain tissue (not shown) was similar to that for brain tumour. Kruskal-Wallis tests of fibroglandular and brain tissue DSCs showed no significant 
difference between experts and a weak significant difference $(\mathrm{p}=0.046)$ between experts for brain tumour DSCs.

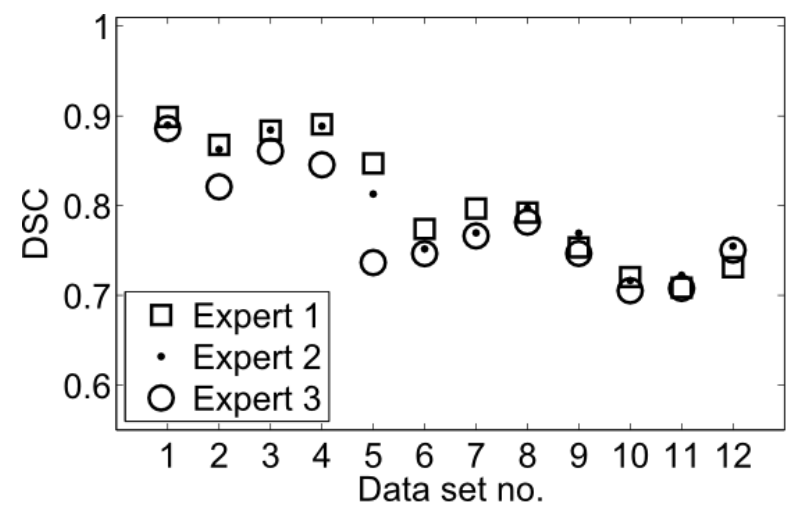

a. Fibroglandular tissue validation, inter-expert variation

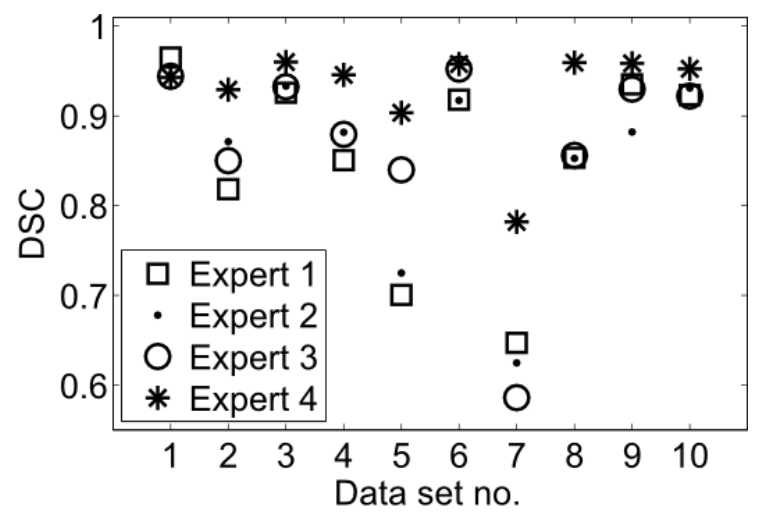

b. Brain tumour validation, inter-expert variation

Figure 5. Inter-expert variation, differences amongst experts in the validation of fibroglandular tissue and brain tumour segmentation are shown in a. and b. respectively.
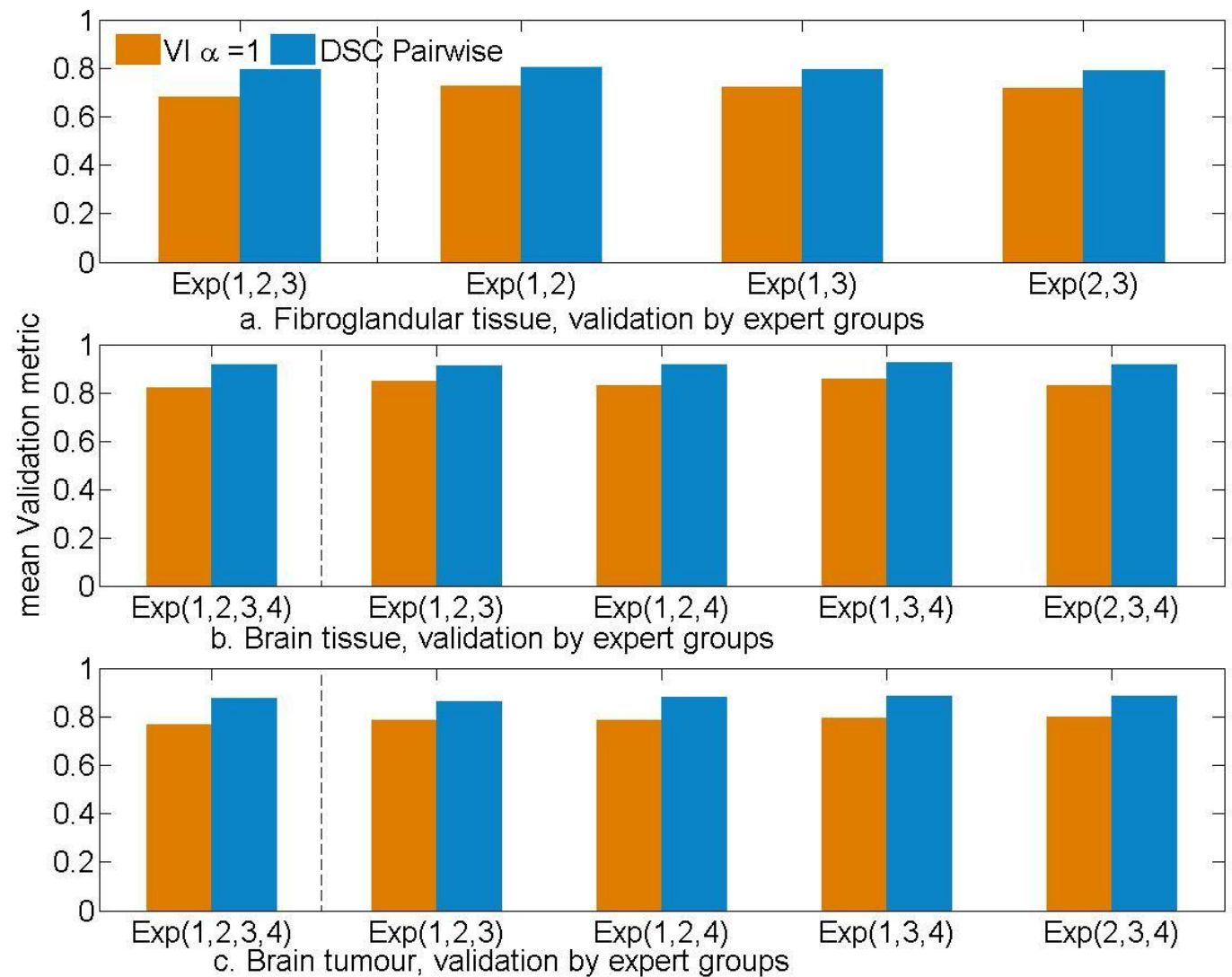

Figure 6. Validation metrics (VI, $\mathrm{DSC}_{\text {pair-wise}}$ ) for the three segmentation structures from clinical data sets: a. fibroglandular tissue; a. brain tissue; c. brain tumour. $\operatorname{Exp}(.$.$) represents the experts used for validation.$ 


\subsection{Effect of number of experts on VI}

Mean validation metrics for each group of experts for the fibroglandular tissue, brain tissue, and brain tumour are presented in figure 6. DSC between observers were similar and therefore we do not expect the VI and $\mathrm{DSC}_{\text {pair-wise }}$ to vary due to variations between observers. Both the mean VI and the mean DSC for the primary group and groups with one less expert outline were similar. There were no significant differences ( $p>0.26)$ between these groups, for all the respective segmentation structures.

\section{Discussion}

In this study, we developed and evaluated the validation index (VI) a metric for validation of segmentation using two or more expert outlines. We constructed VI by taking into account the levels of agreement between expert outlines and adapting the DSC. In case of VI calculation by a single expert outline and also in the case of complete agreement amongst expert outlines, equation (5) reduces to the DSC. The DSC has been shown to be a simple and useful statistical validation metric, which can be applied to studies of accuracy and reproducibility [14]. A study of VI as statistical validation metrics needs to be undertaken using larger datasets. The VI satisfies the following properties:

1. Equals zero if segmentation and expert outlines are disjoint and equals 1 if segmentation and all expert outlines overlap perfectly.

2. The contribution of a point to VI depends on the number of experts who have validated it. (The higher the number of validations, the greater its weight)

3. For two or more cases, where experts' volumes of overlap, expert outlines and overlap of segmentation with the reference standard are identical, the VI is higher for the smaller segmented volume.

4. For two or more cases where segmented volume and overlap of segmentation with the reference standard are identical, the VI is higher for the smaller outlined volumes.

Both a membership value $(\mathrm{m})$ and a weighting parameter $(\mathrm{w})$ are required for the validation index, as 
uncertainty (in the ground truth) in the validation, whereas weighting parameter (w) is needed to make the validation index sensitive to volume of experts' agreement

We introduced the concept of membership value to define the probability of a point being within the ground truth ROI. The basic principle of the proposed membership value can be said to build upon the coverage probability concept [15] which is defined as the probability for a point to be covered by the ROI. This concept has been used for number of applications which include probability-based planning [15,16], construction of the average ROI from the repeat scans [17], and also to measure similarity amongst two or more expert outlines [8]. The exact definitions of this probability are different, for example in Kouwenhoven et al. [8] the value of the probability for a point covered by just one expert outline is zero, in our formulation it has a finite value.

There are a number of other studies in the literature that have used experts' agreement (and disagreement) for validation. Chalana et al. [8] proposed a method to generate a gold standard boundary by averaging multiple expert outlines and derived statistics to validate the algorithmic segmentation. Crum et al [9] used fuzzy set theory, developing a framework for generalized overlap measures which can be used to validate fuzzy segmentation (fractional labelling) and compute a validation metric for multiple images and labels. Warfield et al [10] developed the STAPLE (simultaneous truth and performance level estimation) algorithm for validation of image segmentation using the expectation-maximization framework. Another validation approach evaluates and combines several comparison metrics, such as volumetric overlap and volume difference which can provide complementary information using a scoring system to have a single evaluation criterion $[11,12]$. However, the most appropriate approach for a given validation is dominated by the requirements of the segmentation application. The strength of the VI, a simple overlap measure, developed in this study is that it explicitly incorporates the uncertainty in ground truth in the validation process.

The performance of VI with respect to a common validation metric the $\mathrm{DSC}_{\text {pair-wise }}$ was evaluated using the three simulated cases (dataset A) and two clinical datasets (datasets C and D), see Table 2. VI was found to be more sensitive than the $\mathrm{DSC}_{\text {pair-wise }}$ to the change in agreement between experts for dataset $\mathrm{A}$. In all cases, VI was smaller than the corresponding $\mathrm{DSC}_{\text {pair-wise. }}$. $\mathrm{DSC}_{\text {pair-wise }}$ does 
not take into account the levels of agreement between expert outlines and therefore will tend to overestimate the conformity between outlines and segmentation.

An idealised segmentation case can be analysed to illustrate the benefit of using the knowledge of the experts' agreement. In this example (figure 7), a segmentation (S) was validated using two experts' outlines (Expert 1, Expert 2). All three have the same volume (V) with fifty percent overlap between two experts and seventy five percent $\mathrm{S}$ overlaps with each expert. For this case, $\mathrm{VI}=0.56, \mathrm{DSC}_{\text {pair-wise }}=0.75$. Zijdenbos et al [23] recommends that $\mathrm{DSC}>0.7$ implies good agreement between segmentation and experts, however this can be misleading considering the small level of agreement $\left(V_{2}=0.5 \mathrm{~V}\right)$ amongst experts. Moreover, if $V_{2}$ remains constant but the experts outlines increase, VI will reduce to 0 while $\mathrm{DSC}_{\text {pair-wise }}$ will never be less than 0.38 .

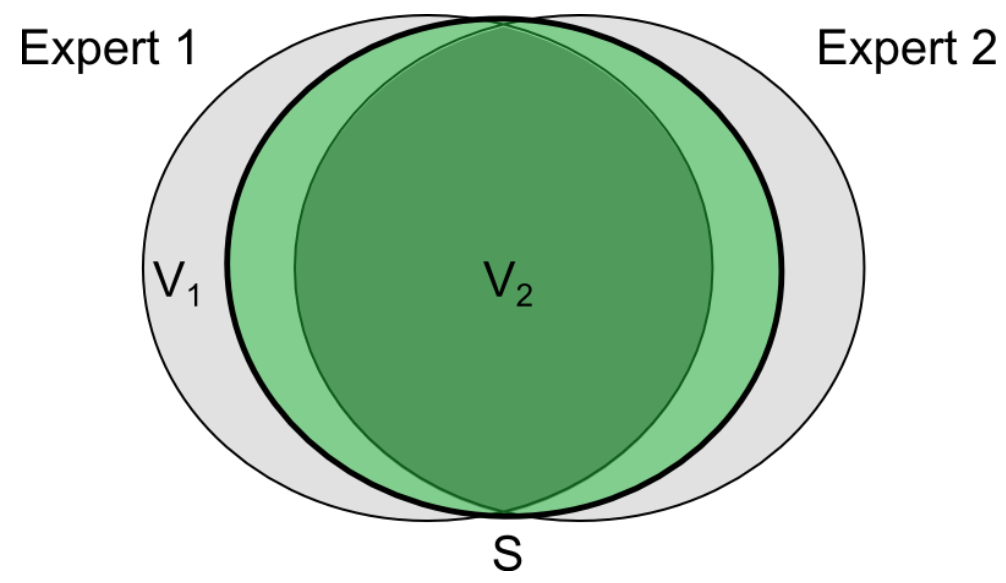

$$
\begin{aligned}
& V_{2}=0.5 \mathrm{~V} \\
& V_{1}=1.0 \mathrm{~V}
\end{aligned}
$$

Expert $1 \cap \mathrm{S} \mid=0.75 \mathrm{~V}$ $\mid$ Expert $2 \cap \mathrm{S} \mid=0.75 \mathrm{~V}$

Figure 7. An example: Validation of algorithmic segmentation (S) by two experts (Expert 1 and Expert 2), all three have same volume $(\mathrm{V})$. In this case $\mathrm{VI}=0.56, \mathrm{DSC}_{\text {pair-wise }}=0.75$. The high value of the $\mathrm{DSC}$ is misleading given the small level of agreement amongst expert outlines. Also with an increase in size of any one of the experts and $\mathrm{V}_{2}$ remaining constant, VI will ultimately reduce to 0 , but $\mathrm{DSC}_{\text {pair-wise }}$ will never become smaller than 0.38 .

Another case can be analysed to illustrate the effect of volume of experts' outline on validation index (VI). In this example (figure 8), segmentation (S) was validated using two experts' outlines (E1, E2). Both expert outlining and segmentation have the volume (V) with ninety percent overlap between two experts and eightyfive percent $\mathrm{S}$ overlap with each expert, for this case $\mathrm{VI}=0.78$. Considering that the segmented volume (S) and overlap of segmentation with the reference standard $\left(\left|V_{k} \cap S_{k}\right| \forall k\right)$ remains constant, and the experts' outlined volume, $\left(\mathrm{V}_{1}\right.$ and $\left.\mathrm{V}_{2}\right)$ are varied $\left(V_{1}=0.2 \mathrm{~V}+\Delta V_{1} ; V_{2}=0.9 \mathrm{~V}+\Delta V_{2}\right)$. For this example, $\mathrm{VI}$ increases with decrease in the volume of experts' outline, as shown in figure 8.b. This change in $\mathrm{VI}$ is more pronounced for change in $V_{2}$ than for change in $V_{1}$, further the change in $\mathrm{VI}$ is linear for small fractional variations in experts' outlined volume as expected from our analytical analysis, given in the appendix A. 


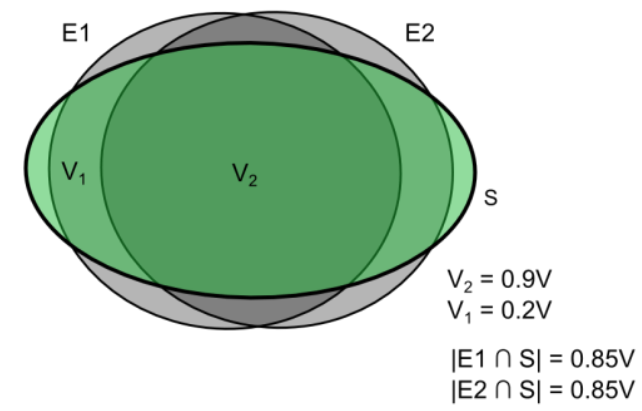

a. Validation of algorithmic segmentation by two experts

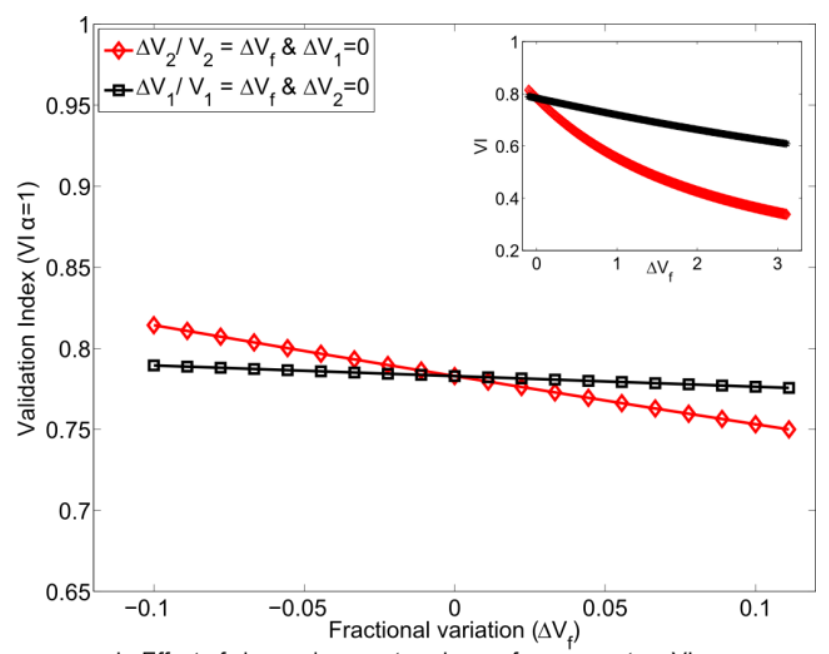

b. Effect of change in experts volume of agreement on $\mathrm{VI}$

Figure 8. An illustration of the effect of variation in experts' agreements on validation index (VI). a. Validation of algorithmic segmentation (S) by two experts (E1 and E2), all having equal volume (V), in this case VI=0.78. b. VI as a function of fractional variation $\left(\Delta V_{f}\right)$ in experts' outlined volume $\left(V_{2}\right.$ and $\left.V_{1}\right)$ under the constraint discussed in the text. Here, VI increases with decrease in the experts' outlined volume as expected by the design of VI. The inset presents variation in VI for larger fractional variation $\left(\Delta V_{f}\right)$.

We have demonstrated the possibility of adapting the VI for the specific requirements in radiotherapy by choosing an appropriate value of control parameter $\alpha$. For a segmentation algorithm that behaves conservatively a higher value of $\alpha$ will yield a higher VI when the algorithm conforms more precisely to volumes of overlap with higher membership values. Conversely, a lower value of $\alpha$ could be chosen if we wish to have a higher value of VI when the algorithm is more inclusive. The choice of control parameter $\alpha$ should be made cautiously depending on the requirements of specific situation. Generally, the use of control parameter $\alpha=1$ might be a good choice because as mentioned earlier this weighting represents an estimate of the probability density function.

It is desirable to have a validation metric that could be used unambiguously to compare studies in which different numbers of expert outlines are used. It has been shown that both VI and the $\mathrm{DSC}_{\text {pair-wise }}$ were unaffected by the number of experts used for the validation (figure 6). $\mathrm{DSC}_{\text {pair-wise }}$ does not utilize the knowledge of the experts' agreement. Moreover, the $\mathrm{DSC}_{\text {pair-wise }}$ cannot be adapted for the specific requirement in radiotherapy planning.

The limitation of the current study is that the VI was evaluated using a limited number of clinical images and idealised test cases. In this regard the VI needs to be evaluated with larger clinical datasets of various regions of interest. The sensitivity of the VI to the levels of expert agreement has been demonstrated qualitatively using three datasets $\mathrm{A} 1, \mathrm{~A} 2$ and $\mathrm{A} 3$, and analytically under certain 
conditions (see Appendix A). However, the systematic sensitivity analysis of the VI needs to be performed to quantitatively assess this sensitivity. Monte Carlo simulations could be used to assess the sensitivity of the VI to the levels of expert agreements. Harris et al [24], in their study to characterize the target volume changes during breast radiotherapy using implanted fiducial markers, used modelling to evaluate the uncertainty in volumes and similarity indices measured for the patient data and calculated corresponding confidence intervals. Gibson et al [25], presented a statistical power calculation model incorporating image registration uncertainty, also used Monte Carlo simulation to assess the accuracy and sensitivity of their model.

\section{Conclusion}

This study developed and evaluated a new metric, validation index, for the validation of algorithmic segmentation using outlines from multiple experts that satisfied the desirable properties. This validation index (VI) could be adapted for specific situations in radiotherapy. VI may be used for a range of validation studies such as an evaluation of algorithmic segmentation methods, and assessment of a new observer in radiotherapy with respect to experienced experts. The VI, a suitable validation metric, presented here is expected to help in the development of automated segmentation methods for radiotherapy treatment planning.

\section{Acknowledgements}

This work was supported by Cancer Research-UK under programme grant reference no C46/A10588 and we would like to acknowledge the NIHR for funding of the biomedical research imaging centre. Prabhjot Juneja is supported by an Institute of Cancer Research PhD studentship.

\section{REFERENCES}

[1] El Naqa I, Yang D, Apte A, Khullar D, Mutic S, Zheng J, et al. Concurrent multimodality image segmentation by active contours for radiotherapy treatment planning. Med Phys 2007;34:4738-4749. 
[2] Klein S, van der Heide UA, Lips IM, van Vulpen M, Staring M, Pluim JPW. Automatic segmentation of the prostate in 3D MR images by atlas matching using localized mutual information. Med Phys 2008;35:14071417.

[3] Isambert A, Dhermain F, Bidault F, Commowick O, Bondiau PY, Malandain G, et al. Evaluation of an atlas-based automatic segmentation software for the delineation of brain organs at risk in a radiation therapy clinical context. Radiotherapy and Oncology 2008;87:93-99.

[4] Thörnqvist S, Petersen JBB, Høyer M, Bentzen LN, Muren LP. Propagation of target and organ at risk contours in radiotherapy of prostate cancer using deformable image registration. Acta Oncol 2010;49:10231032.

[5] Qazi AA, Pekar V, Kim J, Xie J, Breen SL, Jaffray DA. Auto-segmentation of normal and target structures in head and neck CT images: A feature-driven model-based approach. Med Phys 2011;38:6160-6170.

[6] Teguh DN, Levendag PC, Voet PWJ, Al Mamgani A, Han X, Wolf TK, et al. Clinical Validation of AtlasBased Auto-Segmentation of Multiple Target Volumes and Normal Tissue (Swallowing/Mastication) Structures in the Head and Neck. Int J Radiat Oncol Biol Phys 2011;81:950-957.

[7] Dice LR. Measures of the amount of ecologic association between species. Ecology 1945;26:297-302.

[8] Kouwenhoven E, Giezen M, Struikmans H. Measuring the similarity of target volume delineations independent of the number of observers. Phys Med Biol 2009;54:2863-2873.

[9] Cordella LP, Foggia P, Sansone C, Tortorella F, Vento M. Reliability Parameters to Improve Combination Strategies in Multi-Expert Systems. Pattern Anal Applicat 1999;2:205-214.

[10] Juneja P, Harris EM, Evans P, Kirby AM. Evaluation of Breast Tissue Segmentation Methods Using Supine and Prone Computed Tomography Data. Med Phys 2011;38:3745.

[11] Juneja P, Harris EJ, Kirby AM, Evans PM. Adaptive Breast Radiation Therapy Using Modeling of Tissue Mechanics: A Breast Tissue Segmentation Study. Int J Radiat Oncol Biol Phys 2012;84:e419-e425.

[12] Kaus MR, Warfield SK, Nabavi A, Black PM, Jolesz FA, Kikinis R. Automated Segmentation of MR Images of Brain Tumors. Radiology 2001;218:586.

[13] Kaus MR, Warfield SK, Nabavi A, Black PM, Jolesz FA, Kikinis R. Automated Segmentation of Brain Tumors. SPL Brain Tumors Segmentation Image Datasets 2007 Dec . 2007.

[14]Zou KH, Warfield SK, Bharatha A, Tempany C, Kaus MR, Haker SJ, et al. Statistical validation of image segmentation quality based on a spatial overlap index. Academic Radiology 2004;11:178-189.

[15] Stroom JC, de Boer HCJ, Huizenga H, Visser AG. Inclusion of geometrical uncertainties in radiotherapy treatment planning by means of coverage probability. Int J Radiat Oncol Biol Phys 1999;43:905-919.

[16] Baum C, Alber M, Birkner M, Nüsslin F. Robust treatment planning for intensity modulated radiotherapy of prostate cancer based on coverage probabilities. Radiotherapy and Oncology 2006;78:27-35.

[17] Deurloo KEI, Steenbakkers RJHM, Zijp LJ, de Bois JA, Nowak PJCM, Rasch CRN, et al. Quantification of shape variation of prostate and seminal vesicles during external beam radiotherapy. Int J Radiat Oncol Biol Phys 2005;61:228-238.

[18] Chalana V, Kim Y. A methodology for evaluation of boundary detection algorithms on medical images. Medical Imaging, IEEE Transactions on 1997;16:642-652.

[19] Crum WR, Camara O, Hill DLG. Generalized overlap measures for evaluation and validation in medical image analysis. Medical Imaging, IEEE Transactions on 2006;25:1451-1461.

[20] Warfield SK, Kelly HZ, William MW. Simultaneous truth and performance level estimation (STAPLE): An algorithm for the validation of image segmentation. IEEE Trans Med Imag 2004;23:903-921.

[21] Editorial: 3D segmentation in the clinic: A grand challenge II-liver tumor segmentation.: 2008.

[22] A novel adaptive scoring system for segmentation validation with multiple reference masks.: International Society for Optics and Photonics, 2011.

[23]Zijdenbos AP, Dawant BM, Margolin RA, Palmer AC. Morphometric analysis of white matter lesions in MR images: method and validation. IEEE Trans Med Imaging 1994;13:716-724.

[24] Harris EJ, Donovan EM, Yarnold JR, Coles CE, Evans PM. Characterization of target volume changes during breast radiotherapy using implanted fiducial markers and portal imaging. Int J Radiat Oncol Biol Phys 2009;73:958-966.

[25] Gibson E, Fenster A, Ward A. Registration Accuracy: How Good Is Good Enough? A Statistical Power Calculation Incorporating Image Registration Uncertainty. Medical Image Computing and ComputerAssisted Intervention ГÇôMICCAI 2012 2012;643-650. 
